\title{
Transient Angle Obstruction Detected by Anterior-Segment Optical Coherence Tomography and Intraocular Pressure Elevation after Hemodialysis
}

\author{
Mihoko Mochiji Aika Tsutsui Kaoru Manabe Masaki Tanito \\ Department of Ophthalmology, Shimane University Faculty of Medicine, Izumo, Japan
}

\author{
Keywords \\ Hemodialysis - Increased intraocular pressure - Angle-closure glaucoma - Iris bombe . \\ Anterior-segment optical coherence tomography
}

\begin{abstract}
An 80-year-old Japanese man presented with decreased vision in his right eye (OD) after every hemodialysis (HD) session beginning several months previously. His local ophthalmologist prescribed antiglaucoma medications because of high intraocular pressure (IOP) (38 $\mathrm{mm} \mathrm{Hg}$ ) OD 4 months previously; with treatment, the IOP fluctuated between 6 and $34 \mathrm{~mm} \mathrm{Hg}$ OD. When hospitalized, the IOP was measured, and the anterior chamber was observed by anterior-segment optical coherence tomography (AS-OCT) before and after HD. Before HD, the IOP levels were $7 \mathrm{~mm} \mathrm{Hg}$ OD and $8 \mathrm{~mm} \mathrm{Hg}$ in the left eye (OS). AS-OCT showed marked anterior iris bowing due to circumferential posterior synechia OD. The scan showed irido-trabecular contact (ITC) in the nasal angle and not the temporal angle OD. Immediately after HD, the IOP levels were $28 \mathrm{~mm} \mathrm{Hg}$ OD and $12 \mathrm{~mm} \mathrm{Hg} \mathrm{OS;} \mathrm{AS-OCT} \mathrm{showed} \mathrm{ITC} \mathrm{in} \mathrm{the} \mathrm{nasal} \mathrm{and}$ temporal angles OD. Since the iris bombe and HD-induced increase in the ITC were expected to have caused the IOP spike and blurred vision, posterior synechialysis and goniosynechialysis were performed OD. Postoperatively, the iris plane flattened; no IOP spike was recorded, and the blurred vision after HD resolved. At 22 months postoperatively, the IOP was $7 \mathrm{~mm}$ $\mathrm{Hg}$ in both eyes $(\mathrm{OU})$. No deterioration of visual acuity and visual field was recorded during the follow-up period OU. IOP spikes can occur during and after HD because of transient anterior chamber angle obstruction in cases with narrow angles. AS-OCT is useful for detecting minor morphologic changes in the anterior chamber angle during HD.
\end{abstract}

(C) 2021 The Author(s).

Published by S. Karger AG, Basel 
Mochiji et al.: Angle Closure by Hemodialysis

\section{Introduction}

Since 1964 [1], hemodialysis (HD) has been reported to affect intraocular pressure (IOP) [2, 3]. A rapid decrease in plasma osmolality during HD results in an osmotic gradient and causes a fluid shift from the blood to the eyes, causing increased IOP [4]. Removal of water during HD decreases the plasma volume and the concentrations of the plasma proteins increase; this increase in colloid osmotic pressure draws water from the aqueous humor into the plasma, causing decreased IOP [4]. In eyes without aqueous humor outflow resistance, aqueous humor flows out of the angle, but in eyes with outflow resistance, the outflow of aqueous humor is hindered, which is thought to increase the IOP [5]. Although closed or narrow angle was suspected to be a risk for IOP increases after HD [2], few reports have assessed the changes in angle morphology before and after HD $[6,7]$. We report a case of secondary angle closure with repeated marked increases in IOP after HD.

\section{Case Report}

An 80-year-old Japanese man presented to our hospital with decreased vision in his right eye (OD) after every HD session beginning several months previously. He had been undergoing HD 3 times weekly because of chronic renal failure. His ocular history OD included extracapsular cataract extraction and intraocular lens implantation 28 months previously, Klebsiella bacterial keratitis 20 months previously, laser iridotomy (LI) for angle closure 18 months previously, and capsulotomy for after cataract 12 months previously. His local ophthalmologist prescribed topical $0.005 \%$ latanoprost (Xalatan; Pfizer Pharmaceutical, Tokyo, Japan) and 2\% dorzolamide hydrochloride $/ 0.5 \%$ timolol maleate (Cosopt; Santen Pharmaceutical, Osaka, Japan) because of high IOP (38 mm Hg) OD 4 months previously. With medication use, the IOP fluctuated from 6 to $34 \mathrm{~mm} \mathrm{Hg}$ OD. At the initial visit to our hospital, his best-corrected visual acuity (BCVA) and IOP OD were 0.3 and $9 \mathrm{~mm}$ $\mathrm{Hg}$, respectively; the BCVA and IOP in his left eye (OS) were 0.6 and $13 \mathrm{~mm} \mathrm{Hg}$, respectively; the last HD was the day before the visit. During hospitalization, the IOP measurement and anterior chamber observation by anterior-segment optical coherence tomography (AS-OCT) (CASIA 2; Tomey, Nagoya, Japan) were carried out before and after the HD session. The IOPs before the HD in the morning were $7 \mathrm{~mm} \mathrm{Hg}$ OD and $8 \mathrm{~mm} \mathrm{Hg} \mathrm{OS}$. AS-OCT showed marked anterior bowing of the iris due to circumferential posterior synechia OD (Fig. 1a). On this scan, irido-trabecular contact (ITC) was seen at the nasal angle, while no ITC was seen in the temporal angle (Fig. 1b, white double arrow). Immediately after the HD session in the afternoon, the IOPs were $28 \mathrm{~mm} \mathrm{Hg}$ OD and $12 \mathrm{~mm} \mathrm{Hg}$ OS; AS-OCT showed ITC in both the nasal and temporal angles (Fig. 1c, d, double red arrow). The patient was diagnosed with iris bombe due to posterior synechia and transient IOP spikes due to HD-induced intermittent increases in ITC OD. He underwent posterior synechialysis, goniosynechialysis, and a sub-Tenon injection of triamcinolone (MaQaid; Wakamoto Pharmaceutical, Tokyo, Japan) OD. Postoperatively, anterior vitrectomy was performed to remove prolapsed vitreous strands from the anterior chamber. After the surgeries, the iris plane flattened (Fig. 1e, f); no IOP spike was recorded, and the blurred vision after HD resolved. At 22 months postoperatively, the BCVA was 0.6 $\mathrm{OD}$ and $0.4 \mathrm{OS}$, and the IOP was $7 \mathrm{~mm} \mathrm{Hg}$ bilaterally with use of $2 \%$ carteolol hydrochloride (Mikelan LA; Otsuka Pharmaceutical, Osaka, Japan) OD. During the follow-up period, no IOP spike or blurred vision after HD was apparent, and no bilateral deterioration of the visual fields was recorded.

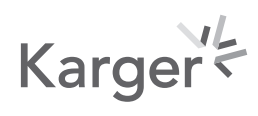




\section{Case Reports in Ophthalmology}

\begin{tabular}{l|l}
\hline Case Rep Ophthalmol 2021;12:761-765 \\
\hline DOI: 10.1159/000513957 & $\begin{array}{l}\text { @ 2021 The Author(s). Published by S. Karger AG, Basel } \\
\text { www.karger.com/cop }\end{array}$ \\
\hline
\end{tabular}
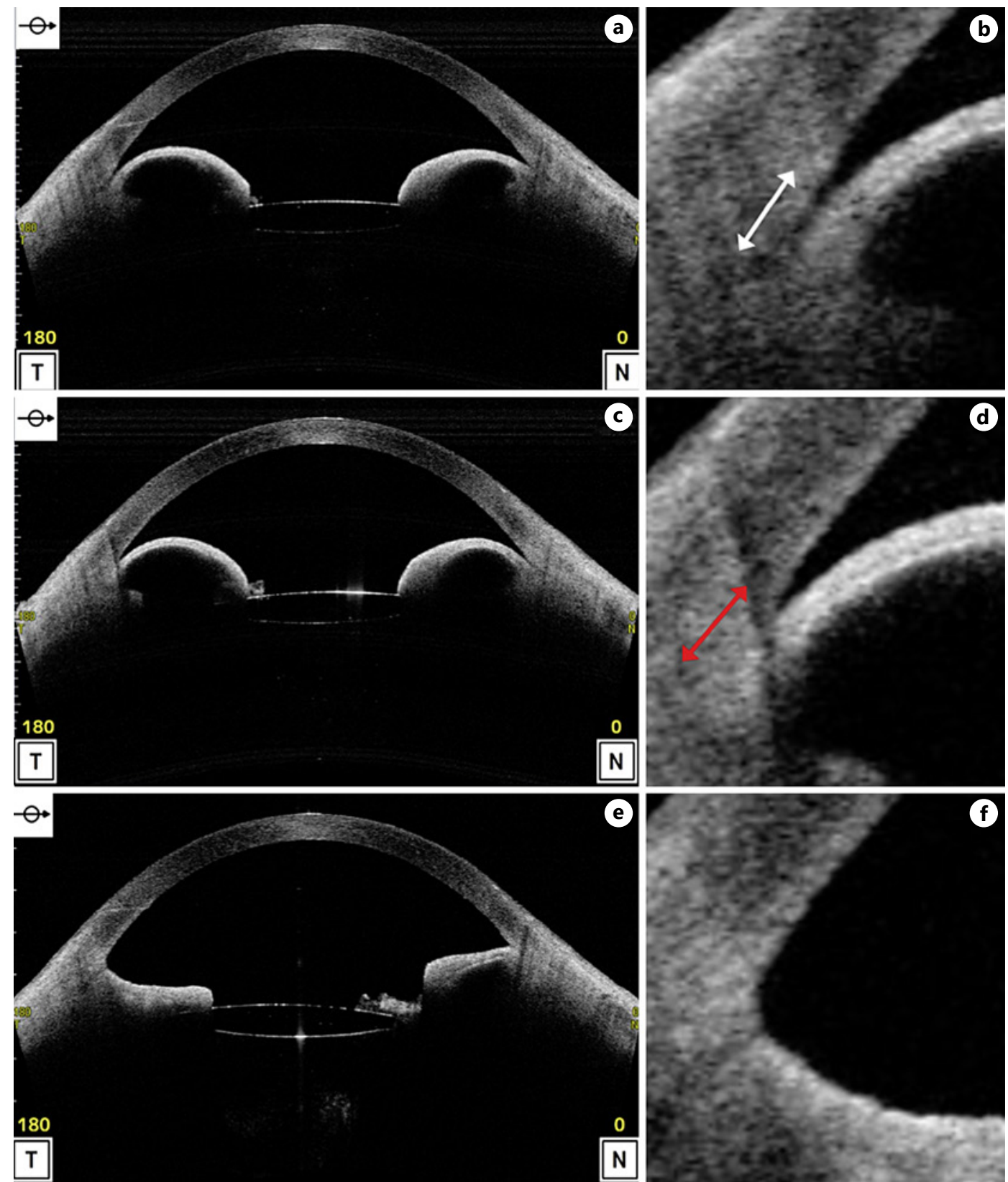

Fig. 1. AS-OCT findings before (a, b) and immediately after (c, d) HD and after posterior synechialysis (e, f). Higher magnification of the temporal angle $(\mathbf{b}, \mathbf{d}, \mathbf{f})$. A narrow but opened angle on the temporal side before HD (b, arrow) closes completely after HD (d, arrow). AS-OCT, anterior-segment optical coherence tomography; HD, hemodialysis.

\section{Discussion/Conclusion}

In the current case, inflammation due to a previous extracapsular cataract extraction and/or bacterial keratitis was thought to be associated with the formation of posterior synechia and iris bombe OD. Although most of the angle was closed OD already, AS-OCT imaging showed no ITC before HD (Fig. 1a, b) and the presence of ITC after HD (Fig. 1c, d) in the temporal angle; this likely explains the IOP change before and after HD in this case. 
The return to a normal IOP thereafter suggested that the ITC OD was transient. Previously, ultrasound biometry assessment in nonglaucomatous subjects, compared with before HD, showed decreases in the anterior chamber depth after HD, while the axial length was unchanged; the pre-HD IOP of $15.26 \mathrm{~mm} \mathrm{Hg}$ did not change after HD [6]. AS-OCT assessment in patients with a nonglaucomatous open angle, compared with before HD, showed decreases in the angle opening distance and trabecular-iris space area after HD; the pre-HD IOP of $17.5 \mathrm{~mm} \mathrm{Hg}$ significantly decreased to $16.2 \mathrm{~mm} \mathrm{Hg}$ after HD [7]. Accordingly, a case with a marked IOP spike after HD due to angle closure seen on AS-OCT images is unique in the literature. Increased inflow during HD in an eye with posterior synechia can explain the forward movement of the iris plane after HD in this case.

Because the previous LI did not work for a long term enough and did not completely relieve the iris anterior bowing, we performed posterior synechialysis and goniosynechialysis. As a result, resolution of the iris bombe was seen on AS-OCT images (Fig. 1e, f), and no IOP spike or blurred vision after HD was apparent for up to 22 months postoperatively.

In conclusion, IOP spikes can occur during and after HD because of transient anterior chamber angle obstruction in patients with narrow angles. AS-OCT is useful for detecting minimal morphologic changes in the anterior chamber angle during HD.

\section{Statement of Ethics}

This study adhered to the tenets of the Declaration of Helsinki. The patient provided written informed consent for publication of this case report and any accompanying pictures.

\section{Conflict of Interest Statement}

The authors have no conflicts of interest for this study.

\section{Funding Sources}

No financial support was provided.

\section{Author Contributions}

M.M., A.T., K.M., and M.T. treated the subject and collected the clinical data. M.M. and M.T. wrote the manuscript, and A.T. and K.M. revised the manuscript. All authors approved the final version of the manuscript. The authors agree to be responsible for all aspects of this work.

\section{Data Availability Statement}

Data relevant to this case is all included in this report. 


\section{References}

1 Sitprija V, Holmes JH, Ellis PP. Changes in intraocular pressure during hemodialysis. Invest Ophthalmol. 1964; 3:273-84.

2 Levy J, Tovbin D, Lifshitz T, Zlotnik M, Tessler Z. Intraocular pressure during haemodialysis: a review. Eye. 2005;19(12):1249-56.

3 Liakopoulos V, Demirtzi P, Mikropoulos DG, Leivaditis K, Dounousi E, Konstas AG. Intraocular pressure changes during hemodialysis. Int Urol Nephrol. 2015;47(10):1685-90.

4 Kilavuzoglu AEB, Yurteri G, Guven N, Marsap S, Celebi ARC, Cosar CB. The effect of hemodialysis on intraocular pressure. Adv Clin Exp Med. 2018;27(1):105-10.

5 Masuda H, Shibuya Y, Ohira A. Markedly increased unilateral intraocular pressure during hemodialysis in a patient with ipsilateral exfoliative glaucoma. Am J Ophthalmol. 2000;129(4):534-6.

6 Gracitelli CP, Stefanini FR, Penha F, Góes MÂ, Draibe SA, Canziani ME, et al. Anterior chamber depth during hemodialysis. Clin Ophthalmol. 2013;7:1635-9.

7 Shin YU, Kim JH, Cho H, Kim DS, Yi J-H, Han S-W, et al. Effect of hemodialysis on anterior chamber angle measured by anterior segment optical coherence tomography. J Ophthalmol. 2019;2019. 